hep-th/0402152

MCTP-04-07

PUPT-2110

\title{
On Black Hole Thermodynamics of 2-D Type 0A
}

\author{
Joshua L. Davis ${ }^{1}$, Leopoldo A. Pando Zayas ${ }^{1}$ and Diana Vaman ${ }^{2}$ \\ 1 Michigan Center for Theoretical Physics \\ Randall Laboratory of Physics, The University of Michigan \\ Ann Arbor, MI 48109-1120 \\ joshuald, lpandoz@umich.edu \\ ${ }^{2}$ Department of Physics \\ Princeton University \\ Princeton, NJ 08544 \\ dvaman@feynman.princeton. edu
}

\begin{abstract}
We present a detailed analysis of the thermodynamics of two dimensional black hole solutions to type $0 \mathrm{~A}$ with $q$ units of electric and magnetic flux. We compute the free energy and derived quantities such as entropy and mass for an arbitrary non-extremal black hole. The free energy is non-vanishing, in contrast to the case of dilatonic 2-d black holes without electric and magnetic fluxes. The entropy of the extremal black holes is obtained, and we find it to be proportional to $q^{2}$, the square of the RR flux. We compare these thermodynamics quantities with those from candidate matrix model duals.
\end{abstract}




\section{Introduction}

Black hole thermodynamics provides a bridge between the classical and quantum aspects of gravitational physics. String theory has achieved moderate success in describing the statistical origin of the thermodynamics of some black holes [1]. The recent proposal for a non-perturbative matrix model description of two-dimensional type 0 string theory $[2,3]$ opens the possibility of providing a statistical description of some of the black holes that appear as solutions to the low energy effective action $[4,5]$.

The thermodynamics of 2 -d black holes is very different from its higher dimensional counterparts. Notions like the area of the horizon are simply lacking. There has been, however, extensive work on 2-d black hole thermodynamics and by now this area is well established [6-13] (see [14] for a comprehensive review).

Our aim in this paper is to compute the thermodynamics of the 2-dimensional black hole of $0 \mathrm{~A}$ string theory with $q$ units of electric and magnetic fluxes. Our analysis yields a robust expression for the entropy of this class of black holes which we proceed to compare with the corresponding results provided by matrix models. Recently [15], the mass of the extremal black hole in 2-d type 0A with $q$ units of electric and magnetic RR fluxes was shown to coincide with the energy of the deformed matrix model proposed by Jevicki and Yoneya [16]. Some other quantities were successfully matched in [17]. Our analysis points, however, to a qualitative connection to another matrix model proposed by Kazakov, Kostov and Kutasov (KKK) [18]. Some recent work concerning the KKK model appear in $[19,20]$

The paper is organized as follows. In section 2 we review the solution under investigation. Section 3 is devoted to the calculation of the ADM mass. Section 4 contains our main results which are explicit expressions for the free energy, entropy and ther-

modynamical mass. We also discuss various limits as a way to gain intuition into the results. In section 5 we compare our results with those provided by matrix models. In section 6 we summarize our results and discuss future directions. We have also included appendix $\mathrm{A}$ where we derive the onshell action and appendix $\mathrm{B}$ where we discuss the possibility of a marginal deformation corresponding to turning on the tachyon field and therefore moving into the $\mu \neq 0$ space in the matrix model side. 


\section{The black hole solution}

The low energy effective action for 2-d type 0A string theory in the presence of RR flux is [3]:

$$
\begin{aligned}
S=\int d^{2} x \sqrt{-g}\left[\frac{e^{-2 \Phi}}{2 \kappa^{2}}\right. & \left(\frac{8}{\alpha^{\prime}}+R+4(\nabla \Phi)^{2}-f_{1}(T)(\nabla T)^{2}+f_{2}(T)+\ldots\right) \\
& \left.-\frac{2 \pi \alpha^{\prime}}{4} f_{3}(T)\left(F^{(+)}\right)^{2}-\frac{2 \pi \alpha^{\prime}}{4} f_{3}(-T)\left(F^{(-)}\right)^{2}+\ldots\right],
\end{aligned}
$$

where $f_{i}(T)$ are functions of the tachyon field $T$. It is convenient to dualize the RR field strengths following [21]. Moreover, in the sector with equal number $q$ of electric and magnetic D0 branes the action reduces to [15]:

$$
S=\int d^{2} x \sqrt{-g}\left[e^{-2 \Phi}\left(c+R+4(\nabla \Phi)^{2}-(\nabla T)^{2}+\frac{2}{\alpha^{\prime}} T^{2}\right)+\Lambda\left(1+2 T^{2}\right) \ldots\right],
$$

where $c=8 / \alpha^{\prime}$ and $\Lambda=-q^{2} /\left(2 \pi \alpha^{\prime}\right)$ and we work in units where $2 \kappa^{2}=1$.

A particularly simple class of solution to the equations of motion corresponds to $T=$ 0 . In this case the action becomes a 2-d dilaton gravity with nontrivial cosmological constant. Black hole solutions to such action have been presented in $[4,5,15]^{1}$.

$$
d s^{2}=l(\phi) d t^{2}+\frac{d \phi^{2}}{l(\phi)}
$$

where

$$
l(\phi)=1-\frac{4}{c} e^{\sqrt{c} \phi}\left(\frac{1}{4} \Lambda \sqrt{c} \phi+m\right),
$$

and the dilaton $\Phi=\sqrt{c} \phi / 2$. For $\Lambda<0$ the generic solution looks like a 2-d version of the Reissner-Nordstrom black hole, that is, these are charged solutions with two horizons [5].

In the region $\phi \rightarrow-\infty$ this solution asymptotes to the linear dilaton solution:

$$
d s^{2}=-d t^{2}+d \phi^{2}, \quad \Phi=\sqrt{\frac{c}{4}} \phi .
$$

The near horizon geometry generically looks like 2-d Rindler space with metric $-x^{2} d t^{2}+$ $d x^{2}$. This can be seen by expanding $l(\phi)$ to first order near the outer horizon and then introducing $x \sim \phi^{1 / 2}$. For extremal black holes the linear term vanishes yielding $l(\phi) \sim$

\footnotetext{
${ }^{1}$ We have rescaled the solution with respect to the standard presentation in the literature $[5,15]$ : $t \rightarrow t \sqrt{\frac{4}{c}}$ and $\phi \rightarrow \phi \sqrt{\frac{c}{4}}$. This rescaling guarantees that the metric asymptotes to the flat metric.
} 
$\phi^{2}$ which means that the near horizon geometry is $A d S_{2}$ with metric $-\phi^{2} d t^{2}+d \phi^{2} / \phi^{2}$ [5]. The fact that the extremal black hole interpolates between "flat space" and $A d S_{2}$ was noted in [22] where this analogy with the D3 brane background was pushed to a proposal for $A d S_{2} / C F T_{1}$.

Even though the solution is obtained with vanishing tachyon and therefore zero Liouville potential we argue in appendix B that there is a marginal deformation in the direction of nonzero $\mu$. The existence of such deformation encourages us to believe that there is a well-defined description of these black holes in terms of a matrix models.

\section{The ADM mass of a 0A 2-d black hole}

The question of mass in 2-d dilaton gravity has been answered in a very general context. In this section we follow an account due to Mann [9] which generalizes previous work of Frolov [12] (see [11] for an alternative approach).

The starting point is an action of the general form

$$
S[g, \Phi]=\int d^{2} x \sqrt{-g}\left[D(\Phi) R+H(\Phi) g^{\mu \nu} \partial_{\mu} \Phi \partial_{\nu} \Phi+V\left(\Phi, \Phi_{M}\right)\right],
$$

where $\Phi_{M}$ denotes other types of matter. In the analysis of [9] $V\left(\Phi, \Phi_{M}\right)$ is restricted to have no metric dependence. The presence of a kinetic term for the tachyon in (2.2) would naturally prevent us from simply borrowing the results of [9]. However, for configurations of constant tachyon the potential is indeed independent of the metric and the result of [9] applies. Note that for a constant tachyon the action (2.2) essentially becomes:

$$
S=\int d^{2} x \sqrt{-g}\left[e^{-2 \Phi}\left(R+4(\nabla \Phi)^{2}+c\right)+\Lambda\right]
$$

and the functions $D(\Phi), H(\Phi), V(\Phi)$ from the generic action (3.1) can be identified as

$$
D(\Phi)=e^{-2 \Phi}, H(\Phi)=4 e^{-2 \Phi}, V=\Lambda+c e^{-2 \Phi} .
$$

A generic action such as (3.1) admits a topologically conserved current $[9,10]$,

$$
S_{\mu}=T_{\mu \nu} \epsilon^{\nu \rho} \partial_{\rho} F
$$

where $T_{\mu \nu}$ is the corresponding stress-energy tensor, provided that

$$
F=F_{0} \int^{\Phi} d s D^{\prime} \exp \left(-\int^{s} d t \frac{H(t)}{D^{\prime}(t)}\right) .
$$


In particular, for us $F$ is proportional to the dilaton $\Phi$. The proportionality constant $F_{0}$ is fixed from the condition that for large $x$

$$
\lim \frac{d F}{d x} \longrightarrow 1
$$

The current (3.4) can be used to define a mass $\mathcal{M}$ independently of the existence of a time-like Killing vector via ${ }^{2} S^{\mu}=\epsilon^{\mu \nu} \partial_{\nu} \mathcal{M}$

$$
\mathcal{M}=F_{0}\left[\int^{\Phi} d s D^{\prime}(s) V(s) \exp \left(-\int^{s} d t \frac{H(t)}{D^{\prime}(t)}\right)-(\nabla D)^{2} \exp \left(-\int^{\Phi} d t \frac{H(t)}{D^{\prime}(t)}\right)\right] .
$$

For the solution at hand we obtain that:

$$
\mathcal{M}=4 F_{0} e^{-2 \Phi}\left[(\nabla \Phi)^{2}-\frac{c}{4}+\frac{1}{2} \Lambda \Phi e^{2 \Phi}\right]
$$

and $F_{0}=1 / \sqrt{c}$. Evaluated on the solution (2.3) we find that the mass is

$$
\mathcal{M}=\frac{4}{\sqrt{c}} m
$$

A similar expression for the mass was obtained in $[5,15]$ and justifies the notation for the constant $m$ in the general solution. We disagree however with the ADM mass expression reported in [15]: $\frac{2}{\sqrt{c}} m+\frac{\Lambda}{2 \sqrt{c}}$. The second term is absent from our evaluation of the ADM mass. We will shortly re-derive the same black hole mass via a thermodynamical analysis.

\section{Black hole thermodynamics}

This section is dedicated to studying the thermodynamics of the solution presented in section 2. We will first discuss the temperature and dilaton charge associated with this class of 2-d black holes before moving on to the free energy and derived quantities.

We will be able to recover from our results the thermodynamics of dilatonic 2-d black holes with vanishing cosmological constant [7]. We have exact results for an arbitrary non-extremal black hole, but for the sake of developing some intuition into the behavior of these black holes we address the near-extremal case separately. Finally,

\footnotetext{
${ }^{2}$ The expression for $\mathcal{M}$ in [9] differs from equation (3.9) by a factor of two. We have fixed this overall coefficient using the ADM mass for the 2-d black hole discussed by Witten in [6]. Our normalization also agrees with the ADM mass of [7] in the case of vanishing RR flux.
} 
being concerned with a possible matrix model description along the lines of [15], we explore the large RR flux limit, which is realized as the near extremal limit on the gravity side.

\subsection{Temperature}

For a metric of the form (2.3), the corresponding temperature can be computed from the condition that the Euclidean counterpart does not have conical singularities near the outer horizon ${ }^{3}$ :

$$
T=\frac{1}{4 \pi}\left|l^{\prime}(\phi)\right|_{\phi=\phi_{h}} .
$$

We need to evaluate the above expression at the largest root of $l\left(\phi_{h}\right)=0$. The location of the horizon is dictated by the equation:

$$
1-\frac{4}{c} e^{\sqrt{c} \phi_{h}}\left(\frac{1}{4} \Lambda \sqrt{c} \phi_{h}+m\right)=0 .
$$

The solution to this equation is given via the Lambert function which by definition satisfies $W(z) \exp (W(z))=z$ :

$$
\phi_{h}=-\frac{4 m}{\Lambda \sqrt{c}}+\frac{1}{\sqrt{c}} W\left(\frac{c}{\Lambda} e^{4 m / \Lambda}\right) .
$$

Equations (4.2) and (4.4) are sufficient to evaluate the temperature of a general nonextremal black hole:

$$
T=\frac{\sqrt{c}}{4 \pi}\left|1+\frac{\Lambda}{c} \exp \left(-4 \frac{m}{\Lambda}+W\left(\frac{c}{\Lambda} e^{4 m / \Lambda}\right)\right)\right| .
$$

To gain intuition into the expression for the temperature, let us consider the extremal and near extremal case.

The extremal black hole has zero temperature. The position of the horizon and its mass are:

$$
\phi_{0}=-\frac{1}{\sqrt{c}}-4 \frac{m_{0}}{\Lambda \sqrt{c}}=\frac{1}{\sqrt{c}} \ln \left(-\frac{c}{\Lambda}\right), \quad m_{0}=-\frac{1}{4} \Lambda\left[1+\ln \left(-\frac{c}{\Lambda}\right)\right] .
$$

The above equations (4.6) imply the mass of the extremal black hole can become negative for large enough RR flux. In two dimensions there is an analogue of Witten's

\footnotetext{
${ }^{3}$ This could be easily seen by introducing $r=l^{1 / 2}$ such that $d r=\frac{1}{2} l^{-1 / 2} l^{\prime} d \phi$ and

$$
d s^{2}=\frac{4}{l^{\prime 2}}\left(d r^{2}+\frac{1}{4} l^{\prime 2} r^{2} d t^{2}\right)
$$
}


proof of the positivity of the ADM mass due to Park and Strominger [23]. Thus, in view of the form for the ADM mass (3.9) and the expression for the extremal black hole (4.6) we conclude that amount of flux has an upper bound:

$$
q^{2}<16 \pi e
$$

For a black hole with ADM mass slightly higher than the extremal mass which correspond to parameters $m$ in the range $m=m_{0}+\delta m$ with $\left|\delta m / m_{0}\right| \ll 1$, the position of the outer horizon becomes:

$$
\phi_{h}=\frac{1}{\sqrt{c}} \ln \left(-\frac{c}{\Lambda}\right)+\frac{2 \sqrt{2}}{\sqrt{c}}\left(-\frac{\delta m}{\Lambda}\right)^{1 / 2}-\frac{4}{3 \sqrt{c}} \frac{\delta m}{\Lambda}+\mathcal{O}\left(\left(-\frac{\delta m}{\Lambda}\right)^{3 / 2}\right) .
$$

Thus, the horizon is pushed outward by adding a small amount of matter. This behavior is as in [6]. Similarly, the temperature corresponding to this near-extremal black hole is:

$$
T=\frac{\sqrt{c}}{\sqrt{2} \pi}\left(-\frac{\delta m}{\Lambda}\right)^{1 / 2}
$$

\subsection{Dilaton Charge}

In two dimensions there is a remarkable ambiguity in the choice of the dilaton charge. Any current of the form, $j_{\mu}=-\epsilon_{\mu}^{\nu} \nabla_{\nu} f(\Phi)$, is conserved by symmetry arguments without the involvement of the equations of motion and is therefore topological. Namely,

$$
\nabla_{\mu} j^{\mu}=-\epsilon^{\mu \nu}\left[f^{\prime \prime}(\Phi) \nabla_{\mu} \Phi \nabla_{\nu} \Phi+f^{\prime}(\Phi) \nabla_{\mu} \nabla_{\nu} \Phi\right]
$$

The right hand side of the previous equation is clearly vanishing, irrespective of the choice of the function $f(\Phi)$.

The topological charge associated with the above current is the flux of this current through a space-like slice, $\Sigma$, which stretches from the horizon to some cut-off wall:

$$
\begin{aligned}
D & =\int_{\Sigma} d \Sigma n^{\mu} j_{\mu} \\
& =-\int_{\phi_{W}}^{\phi_{0}} d \phi \sqrt{g_{\phi \phi}} n^{t} g_{t t} \epsilon^{t \nu} \nabla_{\nu} f(\Phi) \\
& =-\int_{\phi_{W}}^{\phi_{0}} d \phi f^{\prime}(\Phi)
\end{aligned}
$$


We take the canonical choice for $f(\Phi)$ such that the dilaton charge is $D=e^{-2 \Phi}$, that is, the function multiplying the Ricci scalar in the action. This choice facilitates the comparison of our results with other relevant calculations in the literature.

\subsection{Thermodynamic relations}

\subsubsection{The free energy}

In this section we evaluate the on-shell Euclidean action corresponding to the black hole solution (2.3). The action has been derived in appendix $\mathrm{A}$ and is given by:

$$
I_{\text {onshell }}=\int_{\mathcal{M}} \sqrt{g} \Lambda+2 \int_{\partial \mathcal{M}} \sqrt{h} e^{-2 \Phi}\left(K-2 n^{a} \nabla_{a} \Phi\right) .
$$

Our overall strategy is to extract the thermodynamic quantities associated with this solution following the general approach of [24]. We will use a concrete analysis due to $[7]^{4}$ and derive the on-shell action in terms of measurable/observable quantities. The basic setup is that of making physical observations at the wall of a box that serves as the boundary of space-time. At the wall we can measure the value of the dilaton charge $D_{W}$ and the temperature $T_{W}$ there. In terms of these observable variables the free energy, entropy, energy and dilaton chemical potential can be obtained from the on-shell action $I$ as:

$$
F=T_{W} I, \quad S=-\frac{\partial F}{\partial T_{W}}, \quad E=F+T_{W} S, \quad \psi=-\frac{\partial F}{\partial D_{W}} .
$$

\footnotetext{
${ }^{4}$ Another paper which attempts to provide a rather general framework for evaluating the thermodynamical mass and the entropy of a dilaton-gravity solution is [8]. The point of view embraced by the authors of [8] is that the on-shell dilaton-gravity action associated with a static solution can always be expressed as

$$
I_{\text {onshell }}=-\int d t \int_{\text {horizon }}^{\text {spatial infinity }} d x \partial_{x}\left[4 e^{-2 \Phi} l(\Phi) \partial_{x} \Phi+e^{-2 \Phi} \partial_{x} l(\Phi)\right] .
$$

Furthermore, the second term when evaluated at the horizon yields the entropy, and when evaluated at infinity gives the thermodynamical mass. This observation is based on the fact that $I_{\text {onshell }}=$ $\beta F=\beta E-S$. The role played by the first term of the onshell action is to account for the chemical potential associated with the dilaton charge. A difference between this approach and the one we used is that for us the Einstein-Hilbert action is supplemented with a boundary term that ensures that the variational principle is satisfied. Therefore the boundary terms in (4.12) are evaluated on the boundary of the 2-d manifold that is the Euclidean black hole solution, which is to say that the only contribution coming from the boundary terms arises from spatial infinity.
} 
For a metric of the form (2.3) the quantities related to the curvature are

$$
R=-\partial_{\phi}^{2} l, \quad \Gamma_{t t}^{\phi}=-\frac{l}{2} \partial_{\phi} l, \quad \Gamma_{t \phi}^{t}=-\Gamma_{\phi \phi}^{\phi}=\frac{1}{2 l} \partial_{\phi} l
$$

Considering a foliation given by a unit vector in the $\phi$ direction $n^{\phi}=\sqrt{l}$, the extrinsic curvature of the surface (in this case, curve) orthogonal to the foliation is:

$$
K_{t t}=h_{t t} \Gamma_{t \phi}^{t} n^{\phi}=\frac{\sqrt{l}}{2} \partial_{\phi} l, \quad K=\Gamma_{t \phi}^{t} n^{\phi}=\frac{1}{2 \sqrt{l}} \partial_{\phi} l .
$$

With these ingredients the onshell action becomes:

$$
\begin{aligned}
I & =\beta \Lambda\left(\phi_{W}-\phi_{h}\right)+\beta e^{-\sqrt{c} \phi_{W}}\left(-2 l\left(\phi_{W}\right) \sqrt{c}+l^{\prime}\left(\phi_{W}\right)\right) \\
& =\beta \Lambda\left(\phi_{W}-\phi_{h}\right)-\beta \sqrt{c} e^{-\sqrt{c} \phi_{W}}\left(l\left(\phi_{W}\right)+1+\frac{\Lambda}{c} e^{\sqrt{c} \phi_{W}}\right),
\end{aligned}
$$

where $\beta$ is the inverse temperature of the 2 -d black hole. Using the Tolman relation which relates the temperature at the wall $T_{W}$ to the black hole temperature $T$

$$
T_{W}=T \frac{1}{\sqrt{l\left(\phi_{W}\right)}}
$$

where

$$
T=\frac{\sqrt{c}}{4 \pi}\left|1+\frac{\Lambda}{c} D_{h}^{-1}\right|
$$

and by expressing the parameter $m$ of the 2-d black hole solution as a function of the position of the horizon $m\left(\phi_{h}\right)$

$$
m\left(\phi_{h}\right)=\frac{c}{4} D_{h}+\frac{\Lambda}{4} \ln D_{h}
$$

we arrive at the following expression of the on-shell action (4.16):

$$
I_{W}=\frac{1}{T} \Lambda\left(\phi_{W}-\phi_{h}\right)-\frac{D_{W}}{T}\left(1+\frac{T^{2}}{T_{W}^{2}}+\frac{\Lambda}{c D_{W}}\right) .
$$

This expression of the free energy is not yet ready to evaluate the thermodynamic quantities according to (4.13) because it is not expressed exclusively in terms of observable quantities $\left(T_{W}, D_{W}\right)$ and parameters of the theory $(c, \Lambda)$. In particular, we would like to substitute the dilaton charge at the horizon $D_{h}=e^{-2 \Phi_{h}}$ by an alternative expression dependent on the observables $\left(T_{W}, D_{W}\right)$ and $(c, \Lambda)$. This may be achieved 
by using the Tolman relation: from (4.17) and (4.18) we find an expression containing $D_{h}$ as:

$$
T_{W}=\frac{\sqrt{c}}{4 \pi} \frac{1+\frac{\Lambda}{c} D_{h}^{-1}}{\sqrt{1-\frac{D_{h}}{D_{W}}-\frac{\Lambda}{c D_{W}} \ln \frac{D_{h}}{D_{W}}}} .
$$

This equation should be viewed as an equation for the implicit dependence of the dilaton charge at the horizon on the temperature at the wall.

We have thus determined the free energy of the 2-d black hole:

$$
F=-\frac{\Lambda}{\sqrt{c}} \frac{T_{W}}{T} \ln \frac{D_{W}}{D_{h}}-\sqrt{c} D_{W} \frac{T_{W}}{T}\left(1+\frac{T^{2}}{T_{W}^{2}}+\frac{\Lambda}{c D_{W}}\right),
$$

where $T$ and $D_{h}$ should be understood as functions of $\left(T_{W}, D_{W}\right)$ following from (4.18) and (4.21).

\subsubsection{Thermodynamics at zero $R R$ flux}

Let us consider the case of zero cosmological constant, that is, the solution in the absence of RR flux. The solution for the dilaton at the horizon in terms of physical quantities that follows from (4.21) is:

$$
D_{h}=D_{W}\left(1-\frac{c}{16 \pi^{2} T_{W}^{2}}\right) .
$$

This value of the dilaton allows us to identify $m\left(\phi_{h}\right)$ as:

$$
m\left(D_{W}, T_{W}\right)=\frac{c D_{W}}{4}\left(1-\frac{c}{16 \pi^{2} T_{W}^{2}}\right) .
$$

With these ingredients we find the free energy, entropy and energy of the zero RR flux 2-d black hole:

$$
\begin{aligned}
F & =-4 \pi D_{W}\left(T_{W}+\frac{c}{16 \pi^{2} T_{W}}\right) \\
S & =4 \pi D_{W}\left(1-\frac{c}{16 \pi^{2} T_{W}^{2}}\right) \\
E & =-8 \pi D_{W} \frac{c}{16 \pi^{2} T_{W}} .
\end{aligned}
$$

These quantities coincide precisely with the ones obtained in [7] upon the identification of the temperature at the horizon, $T_{c}$ in the notation of [7], with $\sqrt{c} / 4 \pi$. The flat space linear dilaton subtraction regularizes the divergent quantities. In particular, one finds that this solution has vanishing free energy, that the mass (obtained from the regularized energy) coincides with the ADM mass $M=E-E_{\text {flat space }}=8 \pi D_{W} T(1-$ $\left.\frac{T}{T_{W}}\right)=4 \pi T D_{h}$, and that the entropy is $S=M / T$. 


\subsubsection{Extremal black hole}

In this section we extract some of the thermodynamic properties of the extremal black hole. We will approach these quantities by considering a near extremal black hole. In the near extremal limit the leading order solution to (4.21) has to take the form of (4.6), that is

$$
\phi_{h}=\frac{1}{\sqrt{c}} \ln \left(-\frac{c}{\Lambda}\right)+\delta \phi_{h}
$$

We can solve to first order in the leading correction

$$
\delta \phi_{h}=-4 \pi \frac{T_{W}}{c}\left(1+\frac{\Lambda}{c D_{W}}\left(1+\ln \left(-\frac{c D_{W}}{\Lambda}\right)\right)\right)^{1 / 2} .
$$

This is enough to evaluate the free energy

$$
F=\frac{T_{W}}{T}\left(-\frac{\Lambda}{\sqrt{c}} \ln \left(-\frac{c D_{W}}{\Lambda}\right)-\sqrt{c} D_{W}-\frac{\Lambda}{\sqrt{c}}-\Lambda \delta \phi_{h}\right)-\sqrt{c} D_{W} \frac{T}{T_{W}},
$$

where $\delta \phi_{h}$ is given by (4.27) and

$$
\frac{T}{T_{W}}=\left(1+\frac{\Lambda}{c D_{W}}\left[1+\ln \left(-\frac{c D_{W}}{\Lambda}\right)\right]\right)^{1 / 2} .
$$

Note that this ratio is independent of the temperature at the wall. Hence when differentiating the free energy with respect to $T_{W}$ in (4.13) the only term that contributes is the one proportional to $\delta \phi_{h}$. We find that the entropy of the extremal 2-d black hole with RR flux is simply

$$
S=-\frac{4 \pi \Lambda}{c}=\frac{1}{4} q^{2} .
$$

This value of the entropy is natural to identify with the entropy of the extremal black hole with $q$ units of electric and magnetic RR fluxes.

The perspective of working with a solution with non-vanishing cosmological constant can be interchanged with that of discussing a particular solution with (equal) constant electric and magnetic fluxes. The advantage which comes from this latter point of view is that we can now justify choosing to regularize the divergent quantities by subtracting the linear dilaton flat space as a bona fide solution with vanishing fluxes, just as Gibbons-Hawking treated the Reissner-Nordstrom black hole in the thermodynamical approach [24]. Let us now consider the thermodynamical energy, which after a suitable subtraction of the energy of the reference linear dilaton flat space background, we would like to eventually identify with the mass of the black hole. In the large $D_{W}$ 
limit, corresponding to moving the position of the wall to infinity, the energy computed from (4.13) is

$$
E=-2 \sqrt{c} D_{W}-\frac{\Lambda}{\sqrt{c}} \ln D_{W}+\frac{\Lambda}{\sqrt{c}}\left(\ln \left(-\frac{\Lambda}{c}\right)-1\right) .
$$

It is worth saying that the chosen reference background subtraction will remove only the leading divergence from this expression and we were unable to find another subtraction procedure which would remove both divergences at the same time. We discard the second divergence on the basis that it is an infinite volume factor. One remains with a finite part which can be identified with the thermodynamical mass of the near-extremal solution

$$
M=\frac{\Lambda}{\sqrt{c}}\left(\ln \left(-\frac{\Lambda}{c}\right)-1\right)=-\frac{q^{2}}{4 \pi \sqrt{2 \alpha^{\prime}}}\left(\ln \frac{q^{2}}{16 \pi}-1\right) .
$$

This can be seen to coincide with the ADM mass in the extremal case.

Finally, the chemical potential is

$$
\psi=2 \sqrt{c}
$$

\subsubsection{Thermodynamics of an arbitrary non-extremal OA 2-d black hole}

Let us return to the on-shell action of the $0 \mathrm{~A}$ solution with equal number of electric and magnetic fluxes. We saw that the presence of these fluxes manifests as a negative cosmological constant term in the action, and it is this term which yields the only bulk contribution to the on-shell action. To see more distinctly the source of the various terms in the thermodynamical potentials, let us place a marker, a coefficient $\alpha$ in front of the bulk on-shell action. The free energy is then

$$
F=\alpha \frac{\Lambda}{\sqrt{c}} \frac{T_{W}}{T} \ln \frac{D_{W}}{D_{h}}-\sqrt{c} D_{W} \frac{T_{W}}{T}\left(1+\frac{T^{2}}{T_{W}^{2}}+\frac{\Lambda}{c D_{W}}\right), \quad \alpha=-1 .
$$

In the limit where we take the position of the wall to infinity, we have $D_{W} \rightarrow \infty, T_{W} \rightarrow$ $T$, while keeping the temperature of the black hole $T$ (and thus $D_{h}$ ) finite, the free energy becomes

$$
F=-2 \sqrt{c} D_{W}-\frac{\alpha \Lambda}{\sqrt{c}} \ln \left(\frac{D_{W}}{D_{h}}\right)-\frac{\Lambda}{\sqrt{c}}+\mathcal{O}\left(D_{W}^{-1}\right)
$$

Differentiating (4.21) with respect to $T_{W}$ on both sides one obtains an equation for the derivative of the dilaton charge at the horizon with respect to the temperature at the 
wall: $d D_{h} / d T_{W}$. By substituting it into (4.13), we obtain the 2-d black hole energy

$$
\begin{aligned}
E & =\frac{\sqrt{D_{W}\left(c\left(D_{W}-D_{h}\right)+\Lambda \ln \left(D_{W} / D_{h}\right)\right)}}{2 \Lambda c D_{W}-4 c \Lambda D_{h}+2 \Lambda^{2} \ln \left(D_{W} / D_{h}\right)-c^{2} D_{h}^{2}-\Lambda^{2}}\left(-2 \Lambda c D_{W}+3 \Lambda c D_{h}\right. \\
& \left.+\Lambda^{2}(1+\alpha) \ln \left(D_{h} / D_{W}\right)-\alpha \Lambda c D_{h}-\alpha \Lambda^{2}\right)
\end{aligned}
$$

To find the mass of the non-extremal 2-d black hole, we take as usual the limit $D_{W} \rightarrow \infty$ in the expression of the energy, while keeping the value of the dilaton charge at the horizon fixed

$$
E=-2 \sqrt{c} D_{W}+\frac{\alpha \Lambda}{\sqrt{c}} \ln \left(\frac{D_{W}}{D_{h}}\right)-\alpha \sqrt{c} D_{h}-(1+\alpha) \frac{\Lambda}{\sqrt{c}}+\mathcal{O}\left(D_{W}^{-1}\right)
$$

As before, as the wall is pushed to infinity, the energy diverges. The leading divergence - the first term in (4.37) - is canceled by the linear dilaton flat space background subtraction, leaving us with another divergence, proportional to a volume factor $\Lambda \Phi_{W}$. Discarding this term as well, one is left with a finite expression which we identify with the thermodynamical mass $M$ of the 2-d black hole. With the numerical coefficient $\alpha=-1$, we find

$$
M=\sqrt{c} D_{h}+\frac{\Lambda}{\sqrt{c}} \ln D_{h}=\frac{4}{\sqrt{c}} m
$$

that the thermodynamical mass coincides, as expected, with the ADM mass. The entropy expressed in terms of the observables $\left(T_{W}, D_{W}\right)$ is

$$
S=4 \pi D_{h}
$$

with $D_{h}$ given by (4.21). Note that since as the wall of the box is taken to infinity we keep $D_{h}$ fixed, the entropy remains constant, equal to $4 \pi D_{h}$.

It might come as a surprise that we get the same answer for the entropy in terms of the dilaton charge at the horizon for both dilatonic 2-d black holes with vanishing and non-vanishing cosmological constant. This result is in fact quite universal for 2-d black holes, as shown by [13], and our calculation exactly matches the entropy derived by [13]. It is also worth stressing the robustness of the value of the entropy. Although it might not be clear from the explicit calculations, the entropy (as opposed to other thermodynamical quantities) is insensitive with respect to the position of the wall.

Also note that the results at zero RR-flux are directly obtained from our final expressions by taking $\Lambda=0$. It is interesting to notice the role played by the on-shell 
bulk (volume term) action: with $\Lambda=0$ the on-shell action has only boundary terms, which are therefore responsible for the thermodynamical mass $\sqrt{c} D_{h}$; computing first the thermodynamical mass at $\Lambda \neq 0$ as we did in this subsection and then subsequently setting $\Lambda=0$ in the final result, one observes that the thermodynamical mass at vanishing cosmological constant originates entirely in the bulk term of the on-shell action.

Let us summarize our results for the thermodynamics quantities of 2-d black holes, in terms of the dilaton charge at the horizon $D_{h}$, in the following table:

\begin{tabular}{|c||c|c|c|c|c|}
\hline & $M$ & $T$ & $S$ & $F$ & $\psi$ \\
\hline$\Lambda=0$ & $\sqrt{c} D_{h}$ & $\frac{\sqrt{c}}{4 \pi}$ & $4 \pi D_{h}$ & 0 & $2 \sqrt{c}$ \\
\hline \hline$\Lambda \neq 0$ & $\sqrt{c} D_{h}+\frac{\Lambda}{\sqrt{c}} \ln D_{h}$ & $\frac{1}{4 \pi \sqrt{c} D_{h}}\left|c D_{h}+\Lambda\right|$ & $4 \pi D_{h}$ & $\frac{\Lambda}{\sqrt{c}}\left(\ln D_{h}-1\right)$ & $2 \sqrt{c}$ \\
\hline
\end{tabular}

Table 1: Thermodynamic properties of 2-d black holes in type 0A.

\subsubsection{Near extremal thermodynamics}

Given the bound we found for the number of D0 branes (4.7) we cannot try to make contact with the matrix model results where the amount of flux is considered large. Rather a similar limit can be realized in the black hole by considering the near-extremal limit:

$$
\Delta M / M_{0} \equiv \epsilon \ll 1
$$

given that $M_{0}$ is proportional to the flux.

In this limit we arrive at the following expansions:

$$
\begin{aligned}
T & =\frac{\sqrt{2}|\Lambda| \sqrt{1+\ln (-c / \Lambda)}}{4 \pi \sqrt{c}} \sqrt{\epsilon}+\frac{\Lambda(1+\ln (-c / \Lambda))}{6 \pi \sqrt{c}} \epsilon+\ldots \\
S & =4 \pi\left(\frac{|\Lambda|}{c}+\frac{\sqrt{2} \Lambda \sqrt{1+\ln (-c / \Lambda)}}{c} \sqrt{\epsilon}+\ldots\right)
\end{aligned}
$$

By approximating the temperature with the first term in the expansion, we find that the entropy equals the extremal $2 \mathrm{~d}$ black hole entropy plus a correction $\Delta S \propto \Delta M / T$, even though we should caution that the temperature is mass-dependent according to the previous set of equations. Also, the correction to the extremal black hole entropy respects the Cardy formula $\Delta S / S \propto \sqrt{\frac{\Delta M}{M_{0}}}$. Similarly, by defining the free energy with 
respect to the extremal black hole $\Delta F=\Delta M-T \Delta S$, one finds that $\Delta F=-\Delta M / T$, with the temperature of the black hole again given by the leading term in (4.9).

\section{Comparison with matrix models}

Our main motivation for studying the thermodynamics of 2-d black holes in type 0A is the possibility of the existence of a statistical foundation based in matrix models. In this section we will therefore explore the extent to which a connection can be made between the black hole as solutions of the low energy supergravity action and a dual matrix model.

The natural place to start would be the matrix model for type 0A discussed in [3]. The matrix model in question is described by a system of $N$ decoupled non-relativistic fermions moving in two dimensions with angular momentum related to the RR flux $q$

$$
V(\lambda)=-\lambda^{2}+\frac{q^{2}-1 / 4}{l^{2}}
$$

This is the deformed matrix model of Jevicki and Yoneya [16] and has been extensively studied (see for example $[27,25])^{5}$. With the string coupling constant in the deformed matrix model of the order $g_{s} \sim 1 / q$, up to one loop (in the $1 / q$ expansion) the free energy [25] is

$$
\mathcal{F}=-\frac{1}{8 \pi} q^{2} \log \frac{q^{2}}{L^{4}}+\frac{1}{48 \pi}\left[1+(2 \pi T)^{2}\right] \log \frac{q^{2}}{L^{4}} \ldots
$$

where $T$ is the temperature and $L$ is an IR cut-off. The first non-vanishing contribution to the entropy is one-loop

$$
S=-\frac{\pi}{12} T \log \frac{q^{2}}{L^{4}}
$$

In a recent paper [15], it was observed that the ADM mass of the 0A 2-d extremal black hole matches the energy of the ground state of the deformed matrix model. Our thermodynamical analysis reveals that the entropy of the extremal 2-d black hole $S_{\text {extremal }}=4 \pi|\Lambda| / c=q^{2} / 4$ does not match the entropy of the deformed Jevicki-Yoneya matrix model.

Another matrix model considered as a candidate for describing 2-d black holes is the matrix model of Kazakov, Kostov, and Kutasov (KKK) [18]. This matrix model

\footnotetext{
${ }^{5}$ As explained in [3], in the presence of a Liouville potential only one type of brane is physical. That is, the above potential describes a system where only electric or magnetic branes are present. The solution discussed in this paper corresponds to both electric and magnetic charges being turned on, which is possible only for a vanishing tachyon background.
} 
involves summing over all possible $U(N)$ twists around the Euclidean time circle. The partition function can be written in terms of a sum of Gibbs partition functions over $S U(N)$ representations

$$
Z_{N}(\beta, \lambda)=\sum_{r} \int[D \Omega] \chi_{r}\left(\Omega^{\dagger}\right) \exp \left(\sum_{n \in \mathbf{Z}} \lambda_{n} \operatorname{tr}\left(\Omega^{n}\right)\right) \operatorname{Tr}_{r} e^{-\beta H_{r}}
$$

where $\chi_{r}\left(\Omega^{\dagger}\right)$ is the Weyl character, $H_{r}$ is the Hamiltonian in the representation $r$

$$
H_{r}=P_{r} \sum_{k=1}^{N}\left(-\frac{1}{2} \partial_{x_{k}}^{2}-\frac{1}{2} x_{k}^{2}\right)+\frac{1}{2} \sum_{i \neq j} \frac{\tau_{i j}^{r} \tau_{i j}^{r}}{\left(x_{i}-x_{j}\right)^{2}}
$$

and $x_{i}$ are the eigenvalues of the matrix with the inverted harmonic oscillator potential. The matrices $\tau_{i j}^{r}$ are the $S U(N)$ generators. The free energy of the KKK matrix model has the form

$$
F=\frac{1}{2 \pi R}\left(\frac{1}{4}(2-R)^{2} \lambda^{4 /(2-R)}-\frac{R+R^{-1}}{48} \ln \left(\lambda^{4 /(2-R)}\right)+\sum_{h=2}^{\infty} f_{h}(R) \lambda^{4(1-h) /(2-R)}\right)
$$

where $R$ is the radius of the compactified time circle, and is therefore related to the temperature by $2 \pi R=1 / T$. In the critical theory, where the central charge constraint requires $R=\frac{3}{2}$, the genus zero contribution to the free energy is of the order $l^{\frac{2}{(R-2)}} \sim$ $M \sim \frac{1}{g_{s}^{2}}$.

This model has also a large entropy which is assumed to be associated with the non-singlet sector of the matrix theory $[18,29]: S=\beta_{\text {Hagedorn }} M+\ldots$ where $M$ is the black hole mass $M \sim \frac{1}{g_{s}^{2}}$. We have seen that our calculations show that the 0A 2-d black holes have an entropy $S=4 \pi D_{h}$. Since the string coupling is related to the dilaton charge at the horizon by $D_{h}=1 / g_{s}^{2}$, we find that the black hole entropy is precisely of the order $1 / g_{s}^{2}$. It is also interesting to note that the free energy of type 0A 2-d black hole is non-vanishing as opposed to the 2-d bosonic string black hole $(\Lambda=0)$. This suggests that the KKK matrix model might be better suited for describing the 0A 2d black hole.

\section{Conclusion}

We have computed the thermodynamics of 2-d type 0A black holes with equal number of magnetic and electric D0 branes. Quantities like the ADM mass and the temperature 
of the black hole can be computed based on the geometry of the solution. In this paper we have used a thermodynamical approach, based on evaluating the thermal partition function, which provides new information about the solution. We have computed, for the general nonextremal black hole, its entropy, free energy and chemical potential. An interesting observation is that the positivity of the ADM mass implies an upper bound on the D0 brane flux: $q^{2} \leq 16 \pi e$. Our main results are summarized in table (11). In section 5 we have compared our results with some matrix models that are believed to be of relevance for the type of 2-d black holes we discussed. We found that, as opposed to other results quoted in the literature, our results compare unfavorably with the deformed matrix model proposed by Jevicki and Yoneya. In particular, the form of the thermodynamical entropy of the extremal 2-d black hole disagrees with the matrix model result. On the other hand, we find qualitative agreement with the KKK matrix model. In particular, both entropies go as $g_{s}^{-2}$.

The leading term for the entropy of the nonextremal black hole with a large number of D0 branes goes as $q^{2}$. We believe this results captures precisely that the degrees of freedom being described are those of $q$ D0 branes. This result is very similar to its $A d S_{5} / C F T_{4}$ counterpart where the entropy is proportional to $N^{2}$ describing a stack of $N$ D3 branes.

It would be interesting to identify with certainty the matrix model dual to the black hole solutions discussed here since it will provide a microscopic basis for our discussion of the thermodynamics.

\section{Acknowledgments}

It is a pleasure to acknowledge useful discussions with D. Grumiller, A. Jevicki, I. Klebanov, J. McGreevy, R. McNees, D. Oros and H. Verlinde. LAPZ is partially supported by DoE grant DE-FG02-95ER40899. D.V. is supported by DOE grant DEFG02-91ER40671.

\section{A Onshell action}

As it is usual in gravitational Lagrangians, a term with support only on the boundary is needed for a well-defined variational problem. Only the Einstein-Hilbert term needs a compensating boundary term in the action, since it contains second derivatives. The 
variation of this term contains a term with second derivatives of the metric variation. This is

$$
\begin{aligned}
\delta I_{E H}^{(2)}= & \int_{\mathcal{M}} \sqrt{-g} e^{-2 \phi} g^{a b} \delta R_{a b} \\
= & \int_{\mathcal{M}} \sqrt{-g} e^{-2 \phi} \nabla^{a}\left(\nabla^{b} \delta g_{a b}-g^{b c} \nabla_{a} \delta g_{b c}\right) \\
= & \int_{\mathcal{M}} \sqrt{-g}\left[\nabla^{a}\left[e^{-2 \phi}\left(\nabla^{b} \delta g_{a b}-g^{b c} \nabla_{a} \delta g_{b c}\right)\right]\right. \\
& \left.+2 \nabla^{a} e^{-2 \phi}\left(\nabla^{b} \delta g_{a b}-g^{b c} \nabla_{a} \delta g_{b c}\right)\right] \\
= & \int_{\partial \mathcal{M}} \sqrt{-h} e^{-2 \phi} n^{a}\left[\left(\nabla^{b} \delta g_{a b}-g^{b c} \nabla_{a} \delta g_{b c}\right)\right. \\
& \left.+2 \nabla^{b} \phi \delta g_{a b}\right]+ \text { bulk terms } \\
= & -2 \int_{\partial \mathcal{M}} \sqrt{-h} e^{-2 \phi} \delta K .
\end{aligned}
$$

In the last line the second term vanishes since $\delta g_{a b}=0$ on the boundary. In addition the leftover terms can be shown to give $\delta K$ where $K=h^{a b} \nabla_{a} n_{b}$, where $h_{a b}$ is the metric on the boundary. The bulk terms are simply dropped.

In order that the overall variation of the action vanishes, we add an additional boundary term to (4.12) called the Gibbons-Hawking term:

$$
I_{b n d y}=2 \int_{\partial \mathcal{M}} \sqrt{-h} e^{-2 \phi} K
$$

To calculate thermodynamic quantities, we need to evaluate the thermal partition function, which amounts to evaluating the on-shell action. We can write the on-shell action as a volume integral piece plus a boundary term. To do this, use the equation of motion derived by varying with respect to the dilaton:

$$
R+4(\nabla \phi)^{2}+c+4 e^{2 \phi} \nabla^{a}\left(e^{-2 \phi} \nabla_{a} \phi\right)=0
$$

then substitute the above equation of motion into (4.12) to obtain

$$
\begin{aligned}
I_{b u l k} & =\int_{\mathcal{M}} \sqrt{-g}\left[-4 \nabla^{a}\left(e^{-2 \phi} \nabla_{a} \phi\right)+\Lambda\right] \\
& =\int_{\mathcal{M}} \sqrt{-g} \Lambda-4 \int_{\partial \mathcal{M}} \sqrt{-h} e^{-2 \phi} n^{a} \nabla_{a} \phi .
\end{aligned}
$$


Thus the entire on-shell action is

$$
I_{\text {onshell }}=\int_{\mathcal{M}} \sqrt{-g} \Lambda+2 \int_{\partial \mathcal{M}} \sqrt{-h} e^{-2 \phi}\left(K-2 n^{a} \nabla_{a} \phi\right)
$$

\section{B Marginal tachyon deformations}

The need to allow for nontrivial tachyon field, that is, $\mu \neq 0$ in the description of black holes was pointed out by Witten back in [6]. The main reason being that many matrix model results are singular at $\mu=0$. It is, therefore, important to understand the implications of being forced to work at $\mu=0$ in the context of the gravity solutions. In this appendix we consider turning on a small tachyon field in order to shed some light on the structure of the $\mu=0$ region. We thus consider the tachyon which couples to lowest order the other fields as [3]

$$
\int d^{2} x \sqrt{g}\left(e^{-2 \Phi}\left(-(\nabla T)^{2}+\frac{2}{\alpha^{\prime}} T^{2}\right)+2 \Lambda T^{2}\right) .
$$

The equation of motion following from the above action is

$$
\frac{1}{\sqrt{g}} \partial_{\alpha}\left(\sqrt{g} e^{-2 \Phi} g^{\alpha \beta} \partial_{\beta} T\right)+4\left(\frac{1}{\alpha^{\prime}} e^{-2 \Phi}+\Lambda\right) T=0 .
$$

We are interested in solutions of the form $T=T(\phi)$, only depending on the spatial variable. These solutions determine the profile of possible perturbations. We consider the $\phi \rightarrow-\infty$ limit, that is, we would like to find out what happens at infinity to small perturbations of the tachyon field. In this limit the equation becomes

$$
T^{\prime \prime}-\sqrt{c} T^{\prime}+\frac{4}{\alpha^{\prime}} T=0
$$

and therefore

$$
T \sim e^{\lambda_{ \pm} \phi}, \quad \text { where } \quad l_{ \pm}=\frac{\sqrt{c}}{2}\left(1 \pm \sqrt{1-\frac{16}{c \alpha^{\prime}}}\right) .
$$

The remarkable result is that both solutions have positive real part and therefore the tachyon decays at infinity as $\phi \rightarrow-\infty$. This behavior, just as in a similar context discussed in [6], signals the existence of a marginal deformation. This analysis gives us confidence that the solution described in the paper will exist and perhaps retain some of the thermodynamical properties in the presence of a nonzero tachyon field. This raises hope for the existence of a well-defined $(\mu \neq 0)$ matrix model. 


\section{References}

[1] A. Strominger and C. Vafa, "Microscopic Origin of the Bekenstein-Hawking Entropy," Phys. Lett. B 379 (1996) 99 [arXiv:hep-th/9601029].

C. G. . Callan and J. M. Maldacena, "D-brane Approach to Black Hole Quantum Mechanics," Nucl. Phys. B 472 (1996) 591 [arXiv:hep-th/9602043].

[2] T. Takayanagi and N. Toumbas, "A matrix model dual of type 0B string theory in two dimensions," JHEP 0307 (2003) 064 [arXiv:hep-th/0307083].

[3] M. R. Douglas, I. R. Klebanov, D. Kutasov, J. Maldacena, E. Martinec and N. Seiberg, "A new hat for the c $=1$ matrix model," arXiv:hep-th/0307195.

[4] T. Banks and M. O'Loughlin, "Nonsingular Lagrangians for two-dimensional black holes," Phys. Rev. D 48 (1993) 698 [arXiv:hep-th/9212136].

[5] N. Berkovits, S. Gukov and B. C. Vallilo, "Superstrings in 2D backgrounds with R-R flux and new extremal black holes," Nucl. Phys. B 614 (2001) 195 [arXiv:hepth/0107140].

[6] E. Witten, "On string theory and black holes," Phys. Rev. D 44 (1991) 314.

[7] G. W. Gibbons and M. J. Perry, "The Physics Of 2-D Stringy Space-Times," Int. J. Mod. Phys. D 1 (1992) 335 [arXiv:hep-th/9204090].

[8] C. R. Nappi and A. Pasquinucci, "Thermodynamics of two-dimensional black holes," Mod. Phys. Lett. A 7 (1992) 3337 [arXiv:gr-qc/9208002].

[9] R. B. Mann, "Conservation laws and 2-D black holes in dilaton gravity," Phys. Rev. D 47 (1993) 4438 [arXiv:hep-th/9206044].

[10] M. D. McGuigan, C. R. Nappi and S. A. Yost, "Charged black holes in twodimensional string theory," Nucl. Phys. B 375, 421 (1992) [arXiv:hep-th/9111038].

[11] H. Liebl, D. V. Vassilevich and S. Alexandrov, "Hawking radiation and masses in generalized dilaton theories," Class. Quant. Grav. 14 (1997) 889 [arXiv:grqc/9605044].

[12] V. P. Frolov, "Two-Dimensional Black Hole Physics," Phys. Rev. D 46 (1992) 5383. 
[13] J. Gegenberg, G. Kunstatter and D. Louis-Martinez, "Classical and quantum mechanics of black holes in generic 2-d dilaton arXiv:gr-qc/9501017.

[14] D. Grumiller, W. Kummer and D. V. Vassilevich, "Dilaton gravity in two dimensions," Phys. Rept. 369 (2002) 327 [arXiv:hep-th/0204253].

[15] S. Gukov, T. Takayanagi and N. Toumbas, "Flux backgrounds in 2D string theory," arXiv:hep-th/0312208.

[16] A. Jevicki and T. Yoneya, "A Deformed matrix model and the black hole background in two-dimensional string theory," Nucl. Phys. B 411 (1994) 64 [arXiv:hepth/9305109].

[17] U. H. Danielsson, "A matrix model black hole: Act II," arXiv:hep-th/0312203.

[18] V. Kazakov, I. K. Kostov and D. Kutasov, "A matrix model for the twodimensional black hole," Nucl. Phys. B 622 (2002) 141 [arXiv:hep-th/0101011].

[19] X. Yin, "Matrix models, integrable structures, and T-duality of type 0 string theory," arXiv:hep-th/0312236.

[20] T. Suyama and P. Yi, "A holographic view on matrix model of black hole," JHEP 0402 (2004) 017 [arXiv:hep-th/0401078].

[21] D. M. Thompson, "AdS solutions of 2D type 0A," arXiv:hep-th/0312156.

[22] A. Strominger, "A matrix model for AdS(2)," arXiv:hep-th/0312194.

[23] Y. c. Park and A. Strominger, "Supersymmetry And Positive Energy In Classical And Quantum Two-Dimensional Dilaton Gravity," Phys. Rev. D 47 (1993) 1569 [arXiv:hep-th/9210017].

[24] G. W. Gibbons and S. W. Hawking, "Action Integrals And Partition Functions In Quantum Gravity," Phys. Rev. D 15 (1977) 2752.

[25] K. Demeterfi and J. P. Rodrigues, "States and quantum effects in the collective field theory of a deformed Nucl. Phys. B 415 (1994) 3 [arXiv:hep-th/9306141].

[26] S. Carlip, "Near-horizon conformal symmetry and black hole entropy," Phys. Rev. Lett. 88, 241301 (2002) [arXiv:gr-qc/0203001]. 
[27] K. Demeterfi, I. R. Klebanov and J. P. Rodrigues, "The Exact S matrix of the deformed c = 1 matrix model," Phys. Rev. Lett. 71 (1993) 3409 [arXiv:hepth/9308036].

[28] U. H. Danielsson, "A Matrix model black hole," Nucl. Phys. B 410 (1993) 395 [arXiv:hep-th/9306063].

[29] V. A. Kazakov and A. A. Tseytlin, "On free energy of 2-d black hole in bosonic string theory," JHEP 0106 (2001) 021 [arXiv:hep-th/0104138]. 